\title{
Spectra and multiplicities from NA61/SHINE
}

\author{
Szymon Pulawski ${ }^{*}$ \\ for the NA61/SHINE Collaboration \\ Institute of Physics, University of Silesia, Katowice, Poland \\ E-mail: s.pulawski@cern.ch
}

\begin{abstract}
One of the main physics goals of the NA61/SHINE programme on strong interactions is the study of the properties of the onset of deconfinement. This goal is pursued by performing an energy (beam momentum 13A-158A GeV/c) and system size (p+p, p+Pb, Be+Be, Ar+Sc, Xe+La) scan. This paper reviews results and plans of NA61/SHINE. In particular, recently obtained inclusive spectra in inelastic $\mathrm{p}+\mathrm{p}$ and centrality selected $\mathrm{Be}+\mathrm{Be}, \mathrm{Ar}+\mathrm{Sc}$ interactions at the SPS energies will be shown. The energy dependence of quantities inspired by the Statistical Model of the Early Stage (kink, horn and step) shows interesting behavior in $\mathrm{p}+\mathrm{p}$ interactions, which is not described by Monte-Carlo models. Moreover a comparison with $\mathrm{Be}+\mathrm{Be}$ collisions and results from other heavy ion experiments will be performed.
\end{abstract}

Critical Point and Onset of Deconfinement - CPOD2017

7-11 August, 2017

The Wang Center, Stony Brook University, Stony Brook, NY

\footnotetext{
* Speaker.

${ }^{\dagger}$ This work was partially supported by the National Science Centre, Poland (grant 2015/18/M/ST2/00125).
} 


\section{The NA61/SHINE facility}

The layout of the NA61/SHINE detector [1] is presented in Fig. 1. It is a large acceptance hadron spectrometer with excellent capabilities in charged particle momentum measurements and identification by a set of eight Time Projection Chambers as well as Time-of-Flight detectors.

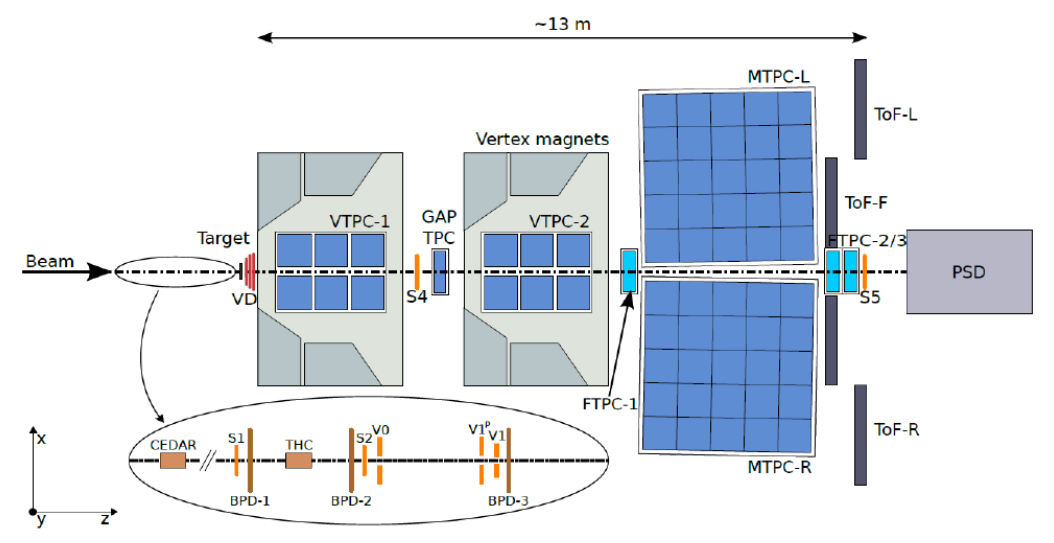

Figure 1: Schematic view of the NA61/SHINE detector system.

The high resolution forward calorimeter, the Projectile Spectator Detector (PSD), measures energy flow around the beam direction, which in nucleus-nucleus reactions is primarily a measure of the number of projectile spectator (non-interacted) nucleons and is thus related to the violence (centrality) of the collision. The PSD measures not only projectile spectators but also produced particles in its acceptance. Thanks to the modular construction of the PSD subsets of modules can be used to optimize centrality selection (only those with anti-correlation between $E_{F}$ and TPC multiplicity) for different energies and systems. Figure 2 presents examples of the module selection and the resulting forward energy $\left(E_{F}\right)$ distributions for Ar+Sc interactions at $19 \mathrm{~A}$ and $150 \mathrm{~A} \mathrm{GeV/c}$.

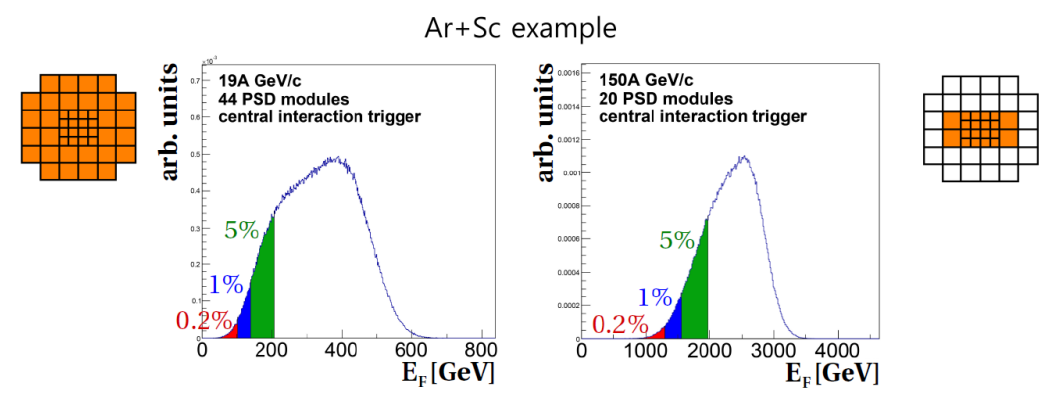

Figure 2: Event (violence) selection is done using the forward energy $\left(E_{F}\right)$ dominated by the energy of projectile spectators. Orange areas on the front face of the PSD indicate the modules used to determine $E_{F}$. Histograms show examples of $E_{F}$ distributions for Ar+Sc interactions at $19 A$ and $150 A \mathrm{GeV} / \mathrm{c}$ with various centrality intervals indicated by colors.

An array of beam detectors identifies beam particles and measures precisely their trajectories. 
Primary and secondary hadron as well as ion beams at $13 A-158 A \mathrm{GeV} / \mathrm{c}$ are used by the experiment.

\section{Charged particle identification}

Final results were obtained for primary particles produced in strong and electromagnetic processes, and are corrected for detector geometrical acceptance and reconstruction efficiency as well as weak decays and secondary interactions. Three analysis methods were used:

- For $\pi^{-}$spectra the $h^{-}$method [2], which is based on the fact that the majority of negatively charged particles are $\pi^{-}$mesons. The contribution of other particles is subtracted using calculations based on the EPOS model [3],

- for $\pi^{+}, \pi^{-}, K^{+}, K^{-}$and $p, \bar{p}$ the $d E / d x$ method, which uses the measured particle energy loss $(d E / d x)$ in the TPC gas to identify particles [4],

- for $\pi^{+}, \pi^{-}, K^{+}, K^{-}$and $p, \bar{p}$ the $t o f-d E / d x$ method, which in addition to the $d E / d x$ information uses time of flight (tof) measurements. Combined tof $-d E / d x$ analysis provides excellent separation of different hadron species close to the mid-rapidity region [4].

\section{3. $\pi^{-}$spectra from the energy and system size scan}

NA61/SHINE measures $\pi^{-}$spectra by the $h^{-}$method in a large acceptance: transverse momentum down to $0 \mathrm{GeV} / \mathrm{c}$, full forward hemisphere. Preliminary rapidity distributions of $\pi^{-}$produced in strong and electromagnetic processes in $\mathrm{Ar}+\mathrm{Sc}$ interactions at 13A, 19A, 30A, 40A, 75A and $150 A \mathrm{GeV} / \mathrm{c}$ are shown in Fig. 3 (left). Rapidity spectra are approximately Gaussian, independent of collision energy and system size. The large acceptance allows to obtain the total multiplicity (sum of data and extrapolation) [5].
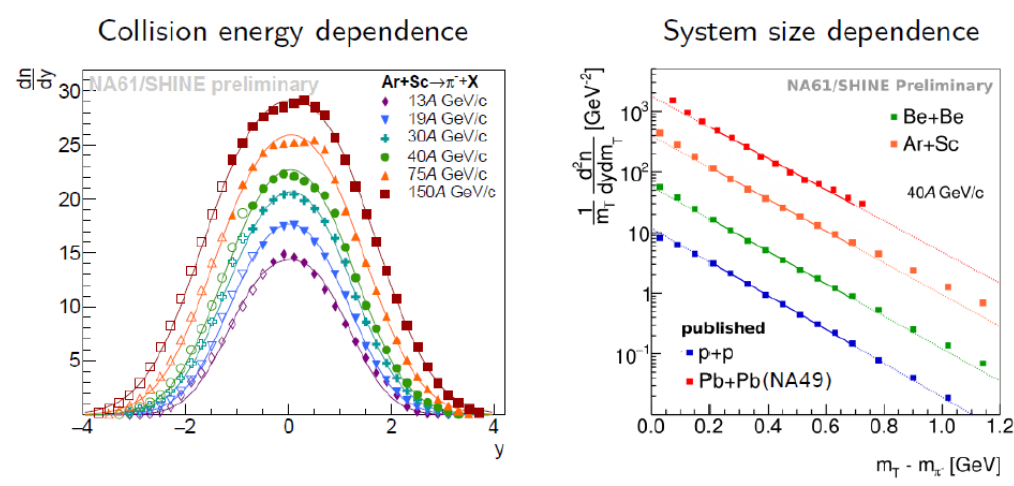

Figure 3: Rapidity distributions of $\pi^{-}$produced in strong and electromagnetic processes in $\mathrm{Ar}+\mathrm{Sc}$ interactions at 13A, 19A, 30A, 40A, 75A and 150A GeV/c (left). Transverse mass distributions of $\pi$ produced in $\mathrm{p}+\mathrm{p}, \mathrm{Be}+\mathrm{Be}, \mathrm{Ar}+\mathrm{Sc}$ and $\mathrm{Pb}+\mathrm{Pb}$ collisions at $40 A \mathrm{GeV} / \mathrm{c}$ around mid-rapidity (right).

Figure 3 (right) presents examples of measured transverse mass $\left(m_{T}\right)$ distributions of $\pi^{-}$produced in $\mathrm{p}+\mathrm{p}, \mathrm{Be}+\mathrm{Be}, \mathrm{Ar}+\mathrm{Sc}$ and $\mathrm{Pb}+\mathrm{Pb}$ collisions at $40 \mathrm{~A} \mathrm{GeV} / \mathrm{c}$ around mid-rapidity. The $m_{T}$ 
spectra in $\mathrm{p}+\mathrm{p}$ collisions are exponential whereas in larger systems for central collisions they deviate from the exponential shape. The shape of $m_{T}$ spectra differs significantly between $\mathrm{p}+\mathrm{p}$ interactions and central A+A collisions. Transverse mass spectra of $\pi^{-}$at mid-rapidity produced in $\mathrm{Be}+\mathrm{Be}, \mathrm{Ar}+\mathrm{Sc}$ and $\mathrm{Pb}+\mathrm{Pb}$ divided by the correspoding results from $\mathrm{p}+\mathrm{p}$ interactions are shown in Fig. 4 at 19A-150A GeV/c. The ratios show a clear system size dependence, but no significant energy dependence for the same system. The change of shape is most likely associated with transverse collective flow.
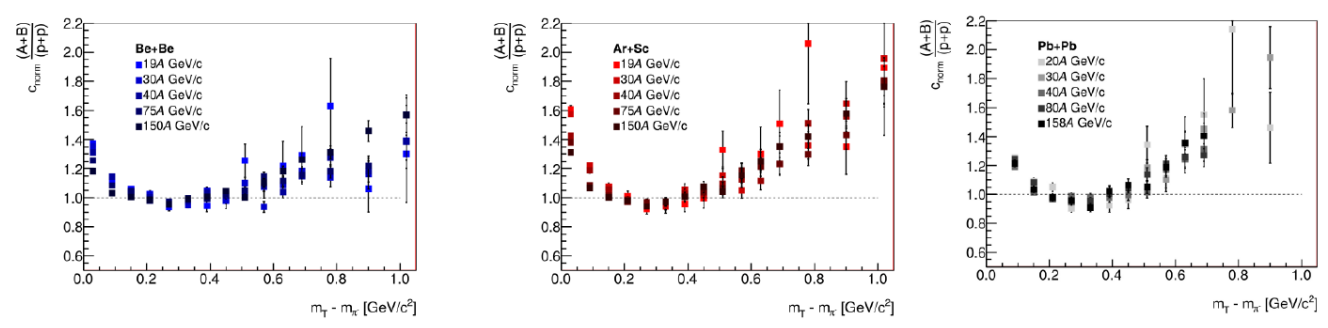

Figure 4: Ratio between the $\pi^{-}$traverse mass $\left(m_{T}\right)$ spectra produced in $\mathrm{Be}+\mathrm{Be}, \mathrm{Ar}+\mathrm{Sc}, \mathrm{Pb}+\mathrm{Pb}$ collisions and corresponding results from $\mathrm{p}+\mathrm{p}$ interactions. Only statistical uncertainties are presented.

\section{Onset of deconfinement}

The Statistical Model of the Early Stage (SMES) [6] predicts a 1st order phase transition to the QGP between top AGS and top SPS energies. In the transition region constant temperature and pressure in the mixed phase and an increase of the number of internal degrees of freedom is expected.

These predictions were confirmed by measurements in central $\mathrm{Pb}+\mathrm{Pb}$ interactions by the NA49 experiment. The ratio of mean total pion multiplicity $\langle\pi\rangle$ and mean number of wounded nucleons $\langle W\rangle$ as function of collision energy ("kink" plot) is presented in Fig. 5. The slope of increase with energy (proportional to the number of internal degrees of freedom) is larger for heavier than for lighter systems at high SPS energies.

A plateau ("step") in the energy dependence of the inverse slope parameter T was observed by the NA49 experiment in $\mathrm{Pb}+\mathrm{Pb}$ collisions for $m_{T}$ spectra of $K^{ \pm}$. It was expected for the onset of deconfinement due to the presence of a mixed phase of hadron gas (HRG) and quark-gluon plasma (QGP). In $\mathrm{p}+\mathrm{p}$ interactions at SPS energies the inverse slope parameter $\mathrm{T}$ of $m_{T}$ spectra show qualitatively similar structure as in central $\mathrm{Pb}+\mathrm{Pb}$ collisions ("step") and such a behavior seems to emerge also in $\mathrm{p}+\mathrm{p}$ and in $\mathrm{Be}+\mathrm{Be}$ reactions, as visible in Fig. 6 . The values of the $\mathrm{T}$ parameter in $\mathrm{Be}+\mathrm{Be}$ collisions are slightly above those in $\mathrm{p}+\mathrm{p}$.

Finally, rapid changes of the ratios $K^{+} / \pi^{+}$at mid-rapidity and $\left\langle K^{+}\right\rangle /\left\langle\pi^{+}\right\rangle$as function of collision energy ("horn") were observed in $\mathrm{Pb}+\mathrm{Pb}$ collisions by the NA49 experiment. These were predicted by the SMES model as a signature of the onset of deconfinement. These two ratios together with new NA61/SHINE results from Be+Be and Ar+Sc collisions are shown in Fig. 7. A plateau like structure is visible in $\mathrm{p}+\mathrm{p}$ interactions. The ratio $\mathrm{K}^{+} / \pi^{+}$at mid-rapidity from $\mathrm{Be}+\mathrm{Be}$ collisions is close to the $\mathrm{p}+\mathrm{p}$ data points. For the three analyzed energies of $\mathrm{Ar}+\mathrm{Sc}$ collisions, the ratio $\left\langle K^{+}\right\rangle /\left\langle\pi^{+}\right\rangle$shows similar dependence on collision energy as in $\mathrm{p}+\mathrm{p}$ interactions. 


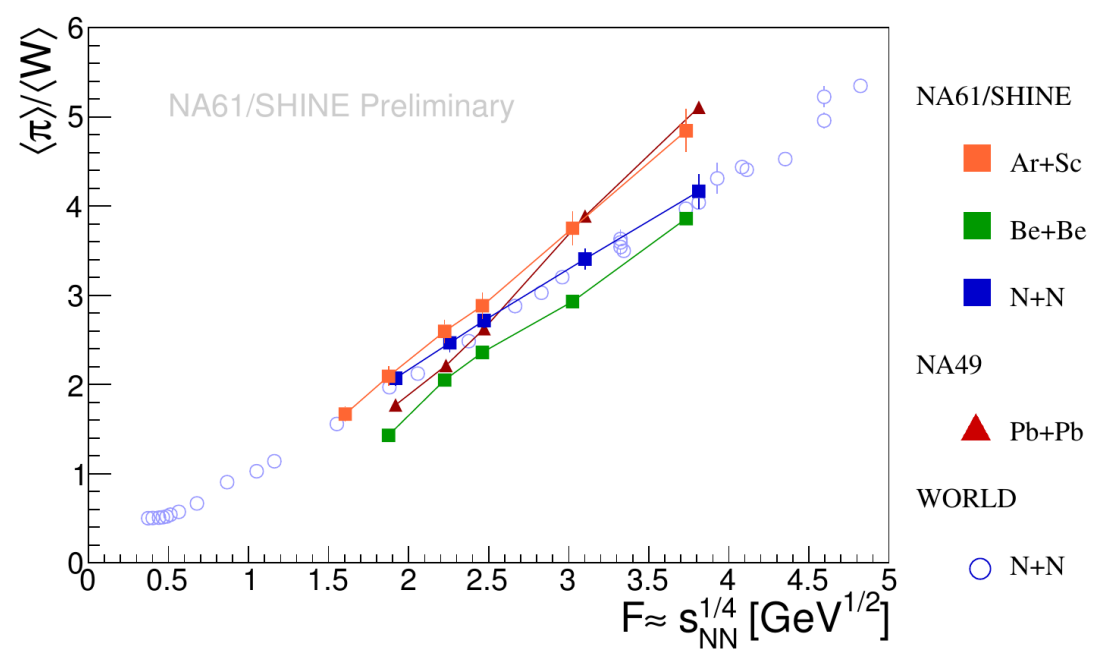

Figure 5: Ratio of mean total pion multiplicity $\langle\pi\rangle$ and mean number of wounded nucleons $\langle W\rangle$ as function of collision energy ("kink" plot). Only statistical uncertainties are shown.
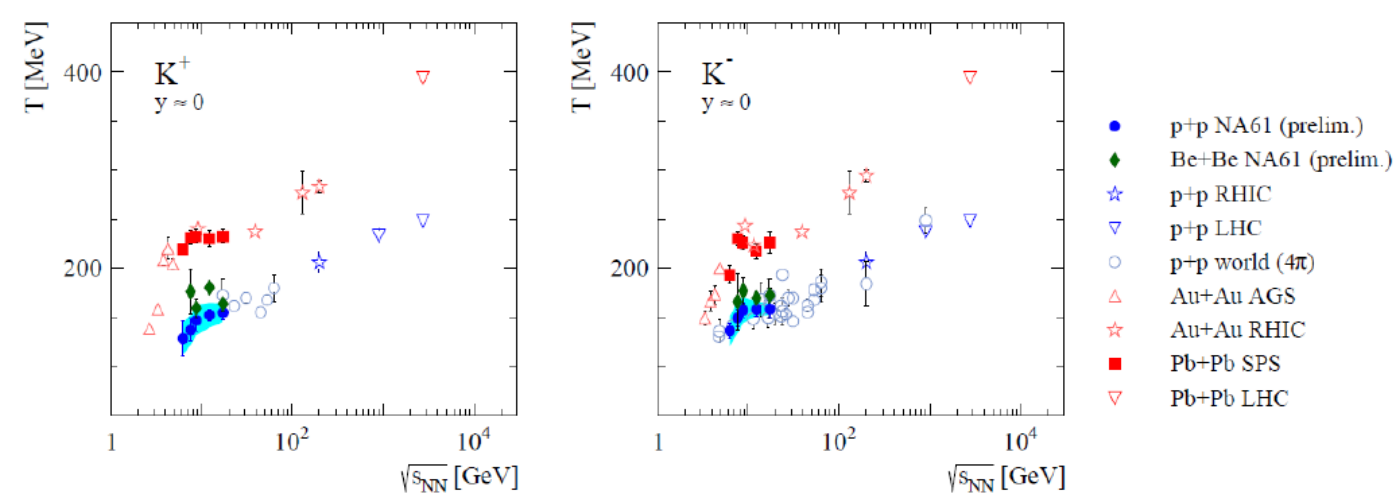

Figure 6: Inverse slope parameter $\mathrm{T}$ of $m_{T}$ spectra of $K^{ \pm}$as function of collision energy. Most results are shown with statistical uncertainties only. For the $\mathrm{p}+\mathrm{p}$ data the shaded band indicates systematic uncertainties.

\section{System size dependence of $K^{+} / \pi^{+}$}

The yield ratio $K^{+} / \pi^{+}$at mid-rapidity at $30 A$ and $150 A \mathrm{GeV} / \mathrm{c}$ as function of the mean number of wounded nucleons $\langle W\rangle$ (system size dependence) is shown in Fig. 8. Surprisingly results for $\mathrm{Be}+\mathrm{Be}$ and $\mathrm{p}+\mathrm{p}$ reactions are very close, independent of collision energy. Comparing to heavier systems the data suggest a jump between light and heavy systems as in the case of multiplicity fluctuations [7].

\section{6. $\phi(1020)$ meson production in $\mathbf{p}+\mathbf{p}$ interactions}

The production of $\phi$ mesons was measured in $\mathrm{p}+\mathrm{p}$ interactions at 40,80 and $158 \mathrm{GeV} / \mathrm{c}$. $\phi$ mesons were identified by their charged kaon decays $\phi \rightarrow K^{+}+K^{-}$using the invariant mass method. The low statistics of the $40 \mathrm{GeV} / \mathrm{c}$ data-set only allowed to extract single differential $d n / d y$ 

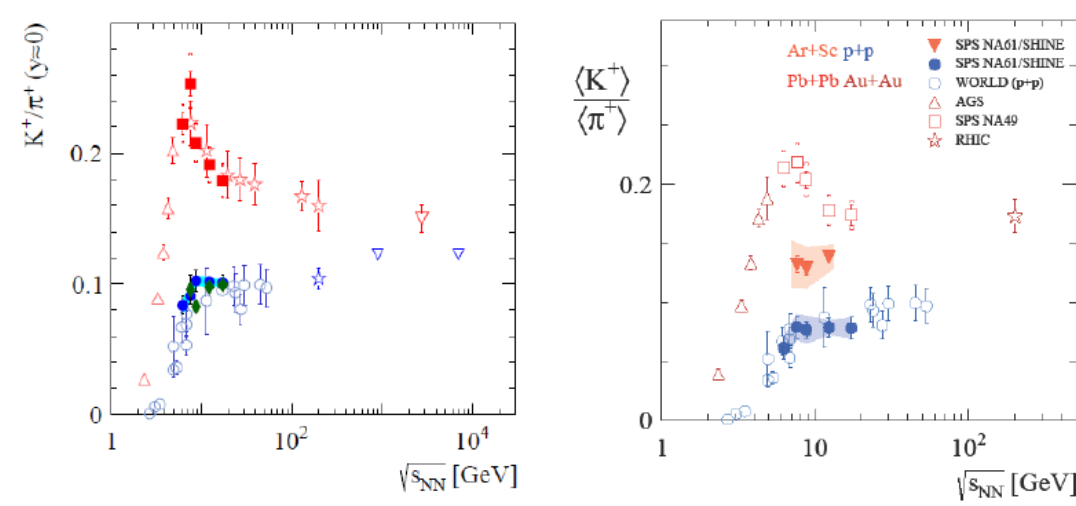

p+p NA61 (prelim.)

Be+Be NA61 (prelim.)

$\mathrm{p}+\mathrm{p}$ RHIC

$\mathrm{p}+\mathrm{p}$ LHC

$\mathrm{p}+\mathrm{p}$ world $(4 \pi)$

$\mathrm{Au}+\mathrm{Au}$ AGS

$\mathrm{Au}+\mathrm{Au}$ RHIC

$\mathrm{Pb}+\mathrm{Pb}$ SPS

$\mathrm{Pb}+\mathrm{Pb}$ LHC

Figure 7: Ratio of yields $K^{+} / \pi^{+}$at mid-rapidity and the ratio of total yields $\left\langle K^{+}\right\rangle /\left\langle\pi^{+}\right\rangle$produced in $\mathrm{p}+\mathrm{p}$, $\mathrm{Be}+\mathrm{Be}$ and $\mathrm{Pb}+\mathrm{Pb}$ collisions as function of collision energy.
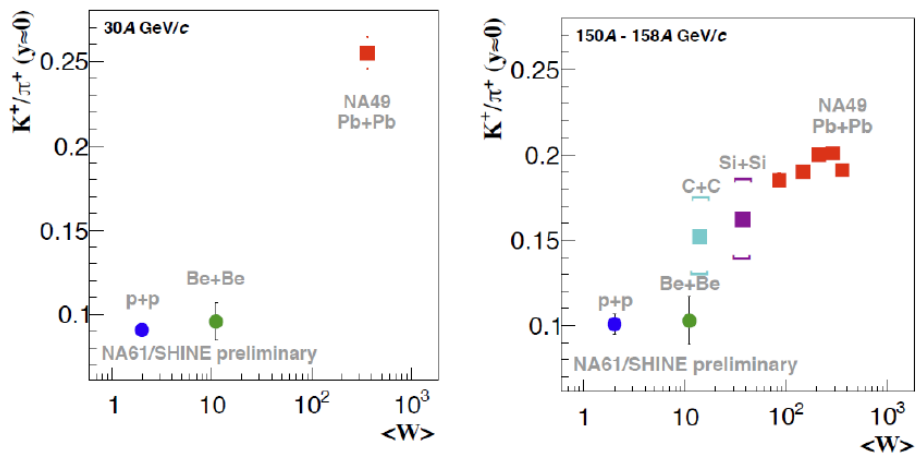

Figure 8: Ratio $K^{+} / \pi^{+}$of mid-rapidity yields in $\mathrm{p}+\mathrm{p}, \mathrm{Be}+\mathrm{Be}$ and $\mathrm{Pb}+\mathrm{Pb}$ collisions as function of the mean number of wounded nucleons $\langle W\rangle$ at $30 A$ and $150 A \mathrm{GeV} / \mathrm{c}$ beam momentum. Only statistical uncertainties are presented.

and $d n / d p_{T}$ spectra in one wide transverse momentum and one rapidity bin, respectively, while at 80 and $158 \mathrm{GeV} / \mathrm{c}$ a double-differential analysis in bins of rapidity and transverse momentum was possible. These are the first measurements of $\phi$ production at 40 and $80 \mathrm{GeV} / \mathrm{c}$ and the first doubledifferential measurements at $158 \mathrm{GeV} / \mathrm{c}$.

Examples of $p_{T}$ spectra are presented in Fig. 9. The spectra are fitted with an exponential function in $m_{T}$ shown by the blue curve. The extracted values of the inverse slope parameter $\mathrm{T}$ at 80 and $158 \mathrm{GeV} / \mathrm{c}$ are in the range $120-160 \mathrm{MeV}$ and decrease with increasing rapidity.

The rapidity spectra of $\phi$ mesons are presented in Fig. 10. Shown are also predictions for the shape of the rapidity spectra from the Epos1.99 [3] (version in CRMC1.6.0 package), Pythia6.4.28 [8] and Urqmd3.4 $[9,10]$ models which are normalized to the integral of the data. All models approximately describe the shape of the spectra. The result of the fit with a single Gaussian to the experimental points is shown by the black curve. The width of the rapidity spectra derived from the fit is compared to the width of the rapidity spectra of $\pi^{-}, K^{ \pm}$in p+p [4] collisions in Fig. 11 (left) and to the width of the rapidity spectra of $\phi$ mesons in $\mathrm{Pb}+\mathrm{Pb}$ [11] collisions in Fig. 11 (right). While 

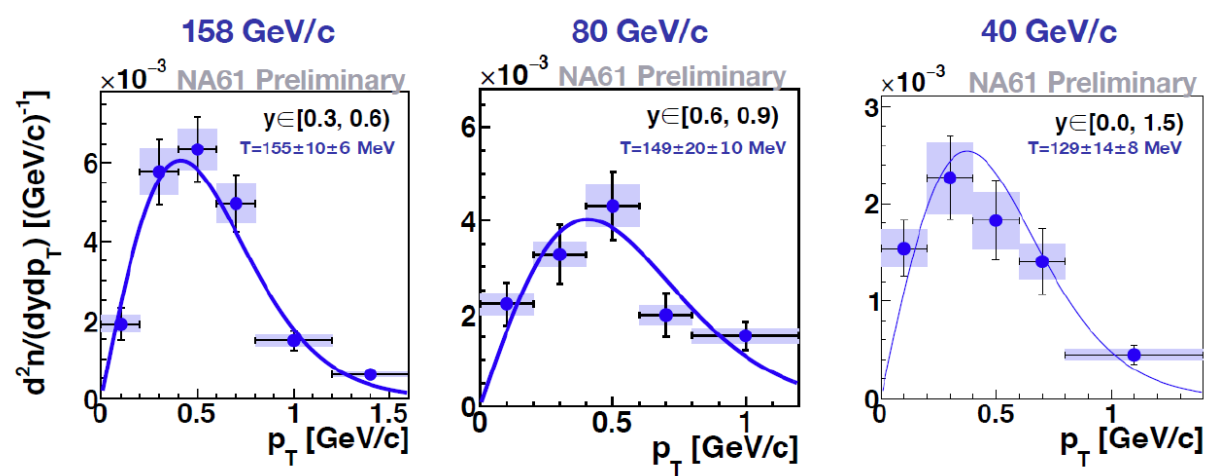

Figure 9: Examples of $p_{T}$ spectra of $\phi(1020)$ mesons produced in inelastic $p+p$ interactions. The systematic uncertainties are shown by the blue shaded boxes while horizontal error bars indicate the width of the $p_{T}$ bins.
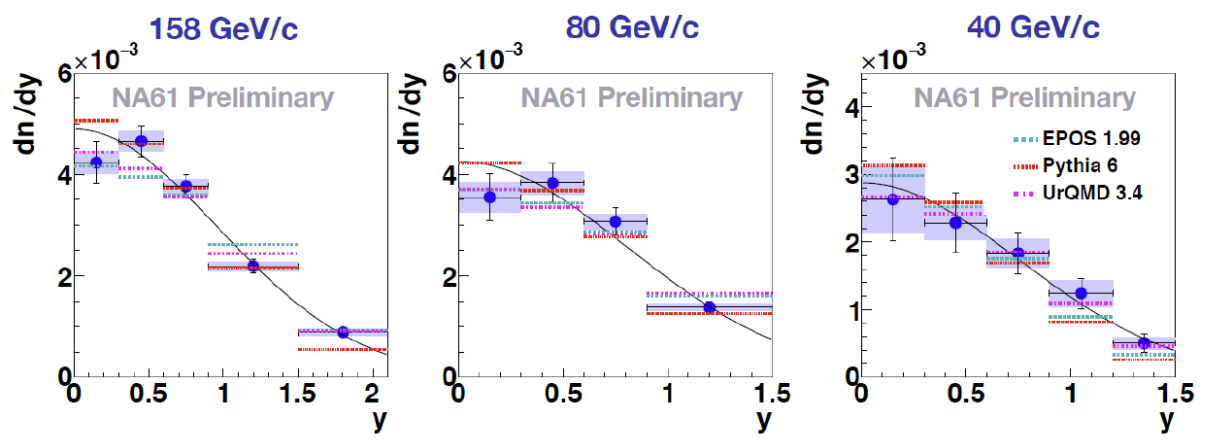

Figure 10: Rapidity spectra of $\phi(1020)$ mesons produced in inelastic $p+p$ interactions. The systematic uncertainties are shown by the blue shaded boxes while horizontal error bars indicate the width of the $p_{T}$ bins.

in $\mathrm{p}+\mathrm{p}$ collisions the $\phi$ follows the trend of the other hadrons, the width of the rapidity distributions of $\phi$ mesons in $\mathrm{Pb}+\mathrm{Pb}$ collisions increases much faster with energy.

\section{NA61/SHINE plans}

The physics program of the NA61/SHINE Collaboration was recently extended by pilot open charm measurements and precise measurement of fluctuations and collective effects in $\mathrm{Pb}+\mathrm{Pb}$ collisions. This extension was possible thanks to the installation of a new precise vertex detector (VD). A small Acceptance version was commissioned in 2015 and test data were recorded in 2016. The vertex fit resolution obtained in the preliminary analysis is on the level of $50 \mu \mathrm{m}$.

Precise measurements of open charm produced in $\mathrm{Pb}+\mathrm{Pb}$ collisions are planned for 20212024. To reach this goal detector upgrades are planned during the Long Shutdown LS2 in 20192020. The readout rate will be increased to $1 \mathrm{kHz}$ by upgrading the TPC readout electronics, a Large Acceptance Vertex Detector (LAVD) will be built based on ALPIDE sensors. Moreover, it is foreseen to increase the intensity and improve the quality of the beam. The predicted number 

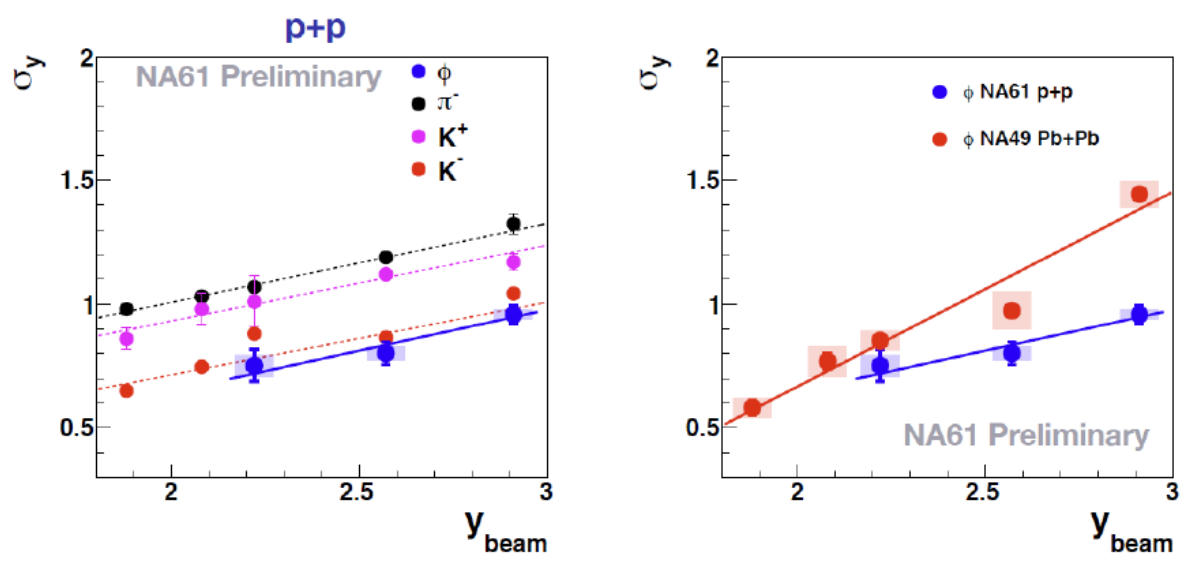

Figure 11: The width of the $\phi(1020)$ meson rapidity spectra as function of energy compared to that of $\pi^{-}$, $K^{ \pm}$in $\mathrm{p}+\mathrm{p}$ [4] interactions (left) and to that of $\phi$ mesons in central $\mathrm{Pb}+\mathrm{Pb}$ collisions [11] (right).

of registered and visible $D^{0}$ decays produced in $\mathrm{Pb}+\mathrm{Pb}$ interactions during 10 days of data taking after 2021 is approximately $4 \cdot 10^{4}$ in $4 \cdot 10^{7}$ events.

\section{References}

[1] N. Abgrall et al. NA61/SHINE facility at the CERN SPS: beams and detector system. JINST, 9:P06005, 2014.

[2] N. Abgrall et al. Measurement of negatively charged pion spectra in inelastic $\mathrm{p}+\mathrm{p}$ interactions at $p_{\text {lab }}=20,31,40,80$ and $158 \mathrm{GeV} / \mathrm{c}$. Eur.Phys.J., C74(3):2794, 2014.

[3] T. Pierog and K. Werner. EPOS Model and Ultra High Energy Cosmic Rays. Nucl.Phys.Proc.Suppl., 196:102-105, 2009.

[4] A. Aduszkiewicz et al. Measurements of $\pi^{ \pm}, K^{ \pm}, p$ and $\bar{p}$ spectra in proton-proton interactions at 20, $31,40,80$ and $158 \mathrm{GeV} / c$ with the NA61/SHINE spectrometer at the CERN SPS. Eur.Phys.J., C77(10):671, 2017.

[5] Maciej Lewicki Pion spectra in Ar+Sc interactions at SPS energies. A. Phys. Pol. B. Supp, 10:645, 2017.

[6] M. Gazdzicki and M. Gorenstein On the early stage of nucleus-nucleus collisions. Acta Phys. Polon., B30:2705, 1999.

[7] M. Gazdzicki Fluctuations and correlation from NA61/SHINE. POS, CPOD 2017.

[8] Sjostrand et al. PYTHIA 6.4 Physics and Manual. JHEP, 05:026 2016.

[9] S. A. Bass et al Microscopic models for ultrarelativistic heavy ion collisions. Prog. Part. Nucl. Phys., 41:255 1998 .

[10] S. A. Bass et al Signatures of quark gluon plasma formation in high-energy heavy ion collisions: A Critical review. J. Phys.,G25:R1 1999.

[11] C. Alt et al Energy dependence of Lambda and Xi production in central $\mathrm{Pb}+\mathrm{Pb}$ collisions at $\mathrm{A}-20$, A-30, A-40, A-80, and A-158 GeV measured at the CERN Super Proton Synchrotron. Phys. Rev., C78:034918 2008. 